\title{
THE AGES OF GLOBULAR CLUSTERS DERIVED FROM BVRI CCD PHOTOMETRY
}

\author{
Gonzalo Alcaino and William Liller \\ Isaac Newton Institute
}

Main sequence photometry has been obtained in the BV system for at least 36 galactic globular clusters (Peterson 1986). One of the most important objectives for these continued researches by several astronomical groups has been that of determining the ages of globular clusters. During the past five years, a general consensus has emerged that the spread in age among the known galactic globular clusters could be as small as 1 billion years; Burstein (1985) suggests that the average age of these objects lies between 14 and 17 Gys.

To obtain reliable ages for globular clusters, two aspects are basic: first, to secure accurate photometry to faint magnitudes; and second, to derive high-quality isochrones. The modern generation of electronic detectors such as the CCD have made improved photometry possible, especially at magnitudes near photographic plate limits. We believe as well that the measurements should be extended to longer wavelenghts, since there now exist BVRI synthetic isochrones provided by the work of VandenBerg and Bell (1985). Using several colors in matching observed and theoretical CMDs to derive cluster ages should be more accurate for several reasons: first, it reduces the importance of blanketing by absorption lines which are an important source of uncertainty in the computation of the isochrones and which are fewer in number in the red and infrared than in the blue. Secondly, the longer baseline in wavelength from the blue to the infrared should make isochrone fitting more accurate since details in the CMDs should stand out more clearly in, for example, (B-I) than in (B-V). Final$1 y$, by having several evaluations of the age of a given cluster, one can intercompare the results and arrive at a more reliable estimate of the accuracy of the final value.

With these considerations in mind, we have embarked on a program of CCD BVRI photometry of globular clusters near enough to the Earth for us to reach below the main sequence turn-off with medium-sized telescopes. We have at this moment completed the reductions of the following 5 globular clusters: NGC 104 (47 Tuc), NGC 2298, NGC 5139 ( $\omega$ Cen), NGC 6121 (M4) and NGC 6362. All the BVRI CCD frames have been obtained with the 1.54 Danish telescope at ESO - La Silla. In order to minimize errors 
produced by photometric transfer and differential extinction we have established photoelectric standards in the same cluster fields with the $1 \mathrm{~m}$ telescope at La Silla. Using the ESO - Max Planck $2.2 \mathrm{~m}$ telescope at La Silla, we have as well obtained BVRI CCD frames of the following eight globular clusters: NGC 1851, NGC 1904, NGC 2808, NGC 3201, NGC 4372, NGC 4590, NGC 5946 and NGC 6139. The data are currently being reduced at La Silla and analyzed at the Isaac Newton Institute. The CMDs of the $\mathrm{V}$ mag versus the color indices, $(B-V),(V-R)$, $(R-I),(V-I)$ and (B-I) have been compared with the isochrones of VandenBerg and Bell (1985). Table I summarizes the basic results of the five clusters so far analyzed. We find that the ages derived for all of them are $17 \pm 1.5 \times 10^{9} \mathrm{y}$, again providing evidence that the globular cluster system is coeval, and that the epoch of the galactic contraction was short. These ages set a lower limit for the age of the universe, and thus an upper limit for the Hubble constant of $\mathrm{H}_{\mathrm{o}}<58 \pm 5 \mathrm{Kms}^{-1}$ assuming $\mathrm{q}_{\mathrm{O}}=0$.

\section{REFERENCES}

Alcaino, G. and Liller W. 1986a Astron. J. 91, 303.

Alcaino, G. and Liller W. 1986b Astron. Astrophys. 161, 61.

Alcaino, G. and Liller W. 1986c, submitted.

Burstein, D. 1985 Publ. Astron. Soc. Pacific 97, 89.

Harris, W. E. and Racine, R. 1979 Ann Rev. Astron. Astrophysics. $17,241$.

Peterson, C. J. 1986, private communication.

VandenBerg, D. A. and Bel1, R. A. 1985 Astrophys. J. Supp1. 58, 561.

TABLE I

SUMMARY OF BASIC RESULTS

\begin{tabular}{|lllllll|}
\hline \hline C1uster & \multicolumn{7}{c}{$\mathrm{V}_{\mathrm{TO}}$} & $\begin{array}{c}\text { Color } \\
\text { Turnoffs }\end{array}$ & $\Delta \mathrm{M}(\mathrm{TO}-\mathrm{HB})$ & $\begin{array}{c}|\mathrm{Fe} / \mathrm{H}| \\
\text { Used for } \\
\text { Isochrones }\end{array}$ & $\begin{array}{c}\text { Age } \\
10^{9} \mathrm{y}\end{array}$ \\
\hline NGC & 104 & $17.60 \pm 0.1$ & $\mathrm{~B}-\mathrm{V}=0.56 \pm 0.02$ & $3.5 \pm 0.2$ & -0.49 & $17.0 \pm 1.5$ \\
NGC & 2298 & $19.50 \pm 0.1$ & $\mathrm{~B}-\mathrm{V}=0.60 \pm 0.02$ & $3.4 \pm 0.2$ & -1.27 & $17.0 \pm 1.5$ \\
NGC & 5139 & $18.30 \pm 0.15$ & $\mathrm{~B}-\mathrm{V}=0.55 \pm 0.03$ & $3.8 \pm 0.2$ & -1.27 & $17.0 \pm 1.5$ \\
NGC & 6121 & $16.80 \pm 0.1$ & $\mathrm{~B}-\mathrm{V}=0.81 \pm 0.02$ & $3.40 \pm 0.2$ & -1.27 & $17.0 \pm 1.5$ \\
NGC & 6362 & $18.75 \pm 0.1$ & $\mathrm{~B}-\mathrm{V}=0.50 \pm 0.02$ & $3.40 \pm 0.2$ & -1.27 & $16.0 \pm 1.5$ \\
\hline
\end{tabular}

